lesion. The main difference between this and the former class of case is that the ventricle here is not necessarily weaker than normal and is, of course, often hypertrophied Speaking broadly, the dilatation of the heart to the right of the sternum is more apt in these cases to equal or exceed in amount that in the second and third left interspaces, and, further, the systolic tricuspid murmur is in these cases more marked and the pulmonary systolic murmur less marked than in the functional cases. Other points of distinction may be gathered from what I have already said.

Symptoms.-The symptoms of dilatation of the right ventricle are mostly of the usual type-viz., breathlessness, palpitation, \&c. There is one symptom, however, which is fairly distinctive of this condition, and that is pain localised to the region of the second or third left costal cartilage. This pain may be fairly acute, but more usually it is dull and aching in character, and is frequently accompanied by a sense of tightness in this region, and there is often in the adolescent class of cases a feeling as if too large a bolus of food had been swallowed and was sticking in the throat at the level of the second left interspace. These sensations are often relieved somewhat when the patient takes a prolonged deep inspiration, and this sighing, voluntary inspiration may often be noted in these cases. In addition, there is sometimes tenderness on percussion over the dilated ventricle, in the second left interspare, and there may even be tenderness on pressure.

Jitiology of dilatation of the right ventricle.-Failure of the right ventricle will result from (1) excessive work thrown apon the normal ventricle-i.e.. absolute overstrain; or 4(2) normal amount of work thrown upon a weakened ventricle-i.e., relative overstrain.

1. Absolute overstrain. - In this class of case the cause of the failure and dilatation of the ventricle is, for the most part, not inherent in the ventricle itself. The most typical examples of this class are those where there is dilatation of the right ventricle as a result of overstrain from excess of athletic exercise or from excess of heavy work, such as lifting heavy weights, in otherwise strong and healthy young men. These cases may be subdivided into-(a) those where the strain upon the right ventricle is indirect and due to the demands of the systemic circulation, as in the cases above mnentioned or in valvular disease of the heart ; and $(b)$ where the strain is due to primary embarrassment of the pulmonary circulation - of this the most typical instance is, of course, emphysema. Pneumonia also produces dilatation from this cause, and I believe that we must also include in this category many, if not most, of the common cases of dilatation of the right ventricle in anæmia and chlorosis. Of course, in the lastnamed cases a prominent feature is the weakness from the anæmia, but I think there are sufficient grounds for believing that there is in addition an abnormal amount of contraction of the pulmonary arterioles caused by the poor state of the blood. This is a subject for physiological experiment. The fact that in suffocation there is a spasm of the pulmonary arterioles. which is removed by nitrite of amyl, as recently pointed sut by Sir George Johnson, is in favour of the above supposition. Two years ago, in my thesis for the degree of M.D., I expressed my belief that the palmonary vaso-motor system ad, in all probability, much to do with the failure of the right side in anæmia, but, unfortunately, ill-health has prevented me from investigating this subject further, except so car as to enable me to say that there seems to be every probawility that the nitrites may prove of considerable value in these cases.

2. Relative overstrain as a cause of dilatation of the right centricle.-As a typical instance of this I may give the dilatation which is apt to occur after the fall of the temperature in rheumatic fever, but more especially when the patient first begins to get up. In such a case I have seen the right ventricle dilate out nearly to the left nipple line in the second left interspace in the conrse of a day or two. The dilatation in cases of anæmia and chlorosis is a'so in part due to weakening of the ventricle from malnutrition, and as another instance may be mentioned the weakening due to many acute febrile conditions.

In conclusion, I may say that there is yet much to learn as to these changes in the right side of the heart, and that there is scope for much carefal clinical study as to the effect of drugs upon the pulmonary circulation, upon the great ' 7 eins, and npon the character and strength of the important expansion movement of the ventricle.

\section{INTESTINAL APPROXIMATION, WITH ESPECIAL REFERENCE TO THE USE OF THE ANASTOMOSIS BUTTON.}

BY J. B. MURPHY, M.D.,

PROFESSOR OF SURGERY AND CLINICAI SURGERY, COLLEGE OF PHYSICIANS AND SURGEONS, CHICAGO, FTC.

Is order to attain good results in intestinal approximation one must consider the means of : (1) securing accurate contact of surfaces; (2) producing speedy and permanent adhesion of the approximated portions ; (3) maintaining an opening sufficiently large for immediate purposes; (4) producing a cicatrix that will not contract to a deleterious degree; and (5) accomplishing all of these in the most simple and rapid manner. The means that have been employed to attain these ends are well classified as follows : (a) suture; (b) suture and mechanical aid; and (c) mechanical means. The microscopical appearances of the line of union at different intervals after operation were fully described both when Czerny-Lembert sutures were employed and when Murphy's button was used, and these tended to show that, though in both cases the union of the several coats was very perfect, yet there was less contraction after the use of the button than after the suture. In comparing the results obtained by the various methods employed in producing intestinal approximation certain conditions, which cannot be accurately estimated, must always cause great doubt as to the trustworthiness of the deductions drawn from statistics alone. These conditions are as follows: (1) cases ending favourably are much more likely to be reported than those that fail ; (2) the condition of the patient before the operation; (3) the pathological conditions for which the operation is performed; (4) the ease with which a means of producing approximation can be applied increases the frequency of its use, especially in desperate cases, where more complicated measures would not be attempted, thereby increasing the chances of a greater percentage of unfavourable results and (5) the dexterity of the operator. With these facts in view, I will proceed with my consideration of the results, beginning with gastro-enterostomy.

Gastro-enterostomy.-The pathological lesions for which this operation is performed are divided into two classes : (1) neoplasms involving the pylorus or duodenum, producing obstruction; and (2) cicatricial contractions occlading the pylorus or duodenum. The most common cause of obstruction is carcinoma of the pylorus. In some cases of this disease the obstruction is the first marked symptom of its presence, while in others it does not appear until the disease is very far advanced and the patient greatly emaciated and cachectic. The disease which produces the obstruction in the first class of cases without excision necessarily terminates fatally, and the operation of gastro-enterostomy in these cases is only for temporary relief. The patient should not be operated upon unless there is a reasonable chance of considerably prolonging life. While the patients may withstand the immediate effects of the operation, after four or five days they become weaker and weaker, until they finally succumb to the general depression, to which has been added the depressing effect of the operation. In 85 cases of gastro-enterostomy for malignant disease there was a mortality of 37.6 per cent., the suture method having a mortality of 42.8 per cent., while when the Murphy button was employed only 1 case died out of 7 (14.3 per cent.).

It has been found that with all other methods except the button cicatricial contraction occurred to a greater degree in gastro-enterostom y by lateral approximation than in any other operation. In the post-mortem records are found reports of complete closure of the opening from cicatricial contraction in five cases. These were all performed by Senn's method with bone-plates-that is, of the twenty-eight cases that survived the immediate effects of the operation five of the number, on whom necropsies were subsequently made, showed a complete closure of the opening. As many of them have not had necropsies it is fair to presume that the percentage of stenosis by this method is very much greater than this. Of these twenty-eight cases the total number in which necropsies were reported is eight. Three of these died before

1 An abstract of a paper real betore the New York Academy of 
the end of four weeks, and the opening was patent; in the remaining five the opening was closed. This would appear to be a very forcible objection to the use of this method in gastro-enterostomy, and should exclude its application. The lateral approximation by means of the button is the only method in which the aperture is produced by a removal of tissue, all other methods depending upon simple incision. The cases of stenosis of the pylorus from other causes than malignant disease are of rare occurrence and are the ones in which it is important to have an approximation made where the element of contraction will not be of moment for an indefinite period of time. The operation that should be performed in this class of cases is complete separation of the end of the duodenum and stomach at the site of constriction. The end of the stomach should be closed with a Czerny-Lembert suture, and the distal end of the duodenum sutured or buttoned to the side of the stomach, thereby avoiding all the disagreeable and dangerous conditions that accompany gastro-enterostomy by lateral approximation.

\section{Pylorectomy with Gastro-enterostomy.}

To Rydygier belongs the honour cf being the first to perform this operation. In Billroth's operation the stomach was sutured from the lesser curvature down until only sufficient opening remained to admit of the insertion of the end of the dnodenum. The end of the duodenum was then united by a Czerny-Lembert suture to the opening in the end of the stomach. Rawdon's operation was practically the same, except that bone-plates were used to approximate the end of the duodenum to the end of the stomach. The time consumed in the operation by the Billroth method was so great (the average being two hours and a half) that all the patients were collapsed before it was completed. There was another danger. It was found that at the junction of the line of suture at the end of the stomach with the circle of suture approximating the duodenum gangrene occurred from the strangulation produced by the suture, and a failure of union resulted, increasing the mortality, already great from shock. To avoid these calamities it was suggested to close the end of the stomach and the end of the duodenum entirely by suture and make a lateral approximation of the stomach to the duodenum or jejunum. This has been performed twentyfour times, with thirteen recoveries (eleven deaths, or 45.8 per cent.). It is safer and more rapid than any means used for the approximation of the end of the duodenum to the end of the stomach. A still better method is the approximation of the end of the duodenum to the posterior wall of the stomach through an incision made for that purpose. The number of this class operated upon by mechanical means is two (anastomosis button); both recovered. The procedure by this method should be as follows. Place a double clamp on the stomach and incise; the stomach between; also a double clamp on the duodenum with incision between. Transfix the mesentery attached to the pyloric portion with a double-threaded needle, and ligate as you would a broad pedicle; excise; apply a continuous Czerny suture to the end of the stomach; remove the clamp and insert a continuous Lembert suture, entirely closing the end of the stomach. Insert the female half of the button in the posterior wall of the stomach as usual, and the male half in the end of the duodenum ; press the button together. The operation can be performed in from thirty to forty minutes. It leaves the end of the stomach securely closed, and the end of the duodenum firmly approximated to the peritoneal surface of the stomach-conditions which are favourable for immediate union. This operation has the following advantages : (1) the danger of shock is very materially lessened by the great reduction of time-i.e., from two hours and a half down to from thirty to forty minutes; (2) the end of the stomach is securely closed; (3) there is a uniform pressure in approximation of the duodenum to stomach; and (4) the danger of peritonitis is lessened, because the peritoneal surface has not been exposed to the atmosphere or manipulated sufficiently to abrade the endothelial layer on its surface, and consequently the likelihood of infection is lessened.

Cholecyst-enterostomy. - Probably the most difficult of all approximations to make by the suture is that of uniting the gall-bladder to the intestinal tract. The results from experimental research by this method were very unsatisfactory, and the verdict of Gaston, Harley, Colzi, De Paige, and others who pursued these experiments industriously was that it was a hazardous operation and one very difficult of execution. The number of operations performed by means of suture up to the present time is twenty-three, with eight deaths, being a mortality of 34.8 per cent.; and the number of opera- tions performed by the anastomosis button for cholelithiasis twenty-three, with twenty-three recoveries. The number of operations in cases of cancer involving the pancreas, the duodenum, the gall-ducts, and the liver is two, with two deaths. The death in one case, reported by Dr. Weir, was due to the disease and not to the method, and oceurred two hours after operation; in the other, a case of my own, to a twisting of the bowel upon itself before the approximation was made, as shown at the necropsy. A com. parison of these results shows that the operation by mechanical means produces most excellent results-ir fact, all that could be desired. It needs no other comment. The explanations for this favourable showing are as follows. 1. The operation can be performed in an extremely short time (in less than twenty minutes) compared to that $\mathrm{b}_{5}$ ? suture, thereby lessening the danger from shock and also of peritoneal infection from prolonged manipalation and $e x$ posure. 2. There is a uniform coaptation of the gall-bladaler to the duodenum, which ensures a union, as there has not beer. in the human subject or in the animals experimented on by this method a single failure of union. The pressule also. prevents hamorrhage, which is one of the dangers of suture, as two patients died from that cause. 3. There is an immediate restoration of bile to the alimentary canal and ar assurance that none of it will escape into the peritoneal cavity at the line of approximation. I believe that the operation of cholecyst-enterostomy by mechanical means will in snitable cases supersede all other operations on the gall-bladder.

Entero-enterostomy.-In treating this subject I will only considex the methods that have been employed and reported in operations in the last six years. These methods I wils arrange as in the previons classification : (1) simple suture, (2) mechanical means, and (3) suture with mechanical aid. The pathological conditions which create the necessity for a resection of a portion of the bowel are of three classes; the order of frequency in which they occur is: (1) strangulated hernia; (2) ileus-intestinal obstruction; (a) strangtulation, (b) obturation; (3) intestinal perforation; (a) traumatic, (b) pathological ; and (4) neoplasms. There will always be a considerable percentage of mortality from the intrinsic dangers of the pathological conditions, aside from the defects of the means employed for their relief. Thezefore the problems to be solved are: (1) how can we reduee the intrinsic dangers of the disease? and (2) how can we minimise the difficulty and danger of the operative procedure, per se? In order to decide the first problem-that is, the intrinsic danger-the subject of diagnosis must be considered, as the dangers of the disease depend in a large measure upon the failure of early diagnosis. This is particalarly true of ileus, and I feel constrained to further emphasise ir connexion with this subject the imperative importance of early and accurate diagnosis, as the result in operations of this class depends more than in any other upon an eari recognition of the pathological condition. The difficulty of making a positive diagnosis of the pathological lesion producing the obstruction has been materially diminished by our modern advancements, but is not generally pnderstood or practised. This necessarily increases the danger to the patient, as the operation is postponed until extreme symptoms manifest themselves There is only one way to obtain good results in ileus-that is, by early diagnosis and operation. In this we must consider every case of ileus as we would an acute strangulatea hernia. We know that an early operation for strangulated hernia admits of a much more favourable prognosis than one which is delayed until the intestine is gangrenous. How much more important is it that the early operation should be per formed in internal strangulation where a gangrene would take place within the peritoneal cavity. Ixpectant treat ment in abdominal lesions is only justifiable when the diagnosis of intestinal obstruction is positively exeluded The only rational outcome to expect from so-called conservative treatment of ileus is death. The differential diagnosis of intestinal obstruction is not very difficult to make if the symptoms have not been masked by the use of opiates.

There are two symptoms, upon which great reliance can be placed, for the differential diagnosis between ilens and perforative peritonitis. 1. In the early stage of perforative peritonitis there is an elevation of temperature; in intestinal obstruction no elevation of temperature. 2. In cerforative: peritonitis there is a paralysis of peristalsis over the inflamed area; in intestinal obstruction there is a very great increase in the peristalsis, which continues until necrosis of the bowel takes place or a peritonitis is developed. Frequent 
arscultation of the abdomen as an aid to diagnosis of intestinal obstruction is of as much value as auscultation of the chest in arriving at a diagnosis in pulmonary diseases. With the stethoscope, or ear, upon the abdomen in the early stage of intestinal obstruction, there is heard a constant rumbling and rolling of the contents of the intestine and manifestations of stormy peristaltic action. These manifestations are most marked at the seat of obstruction, and, indeed, the location - of the obstruction can frequently be ascertained by these signs alone. If this peristaisis should momentarily subside, it can be brought on with increased violence by manipulation of the abdomen. A dose of opium will suppress the peristaltic action of the intestine for hours, while a chloroform narcosis does not interfere with it. Therefore no opiates should be given in acute lesions in the peritoneal cavity until the question of diagnosis is decided. In ileus, where active peristalsis has subsided, and cannot be reproduced by pressure, it is an indication that peritonitis has ensued and the pregnosis of the operation is proportionately grave. A symptom of importance in obstruction of the bowel is a very circumseribed dulness near or at the seat of obstruction, due to accumalation in the proximal portion of the bowel ( $8 \mathrm{chede}$, Schlange) and a meteoric distension of the strangulated loop itself, due to obstructed circulation and decomposition of its contents, producing a rapid, gaseous distension and complete paralysis of the wall (Von Wahl). Kader's experiments show that the distension of the strangulated loop is not due to gas and intestinal contents forced into the loop from the proximal side, as was formerly supposed, but is principally due to dissurbances of the circulation in the intestinal wall, and consists of $a$ uniform expansion of the entire intestine, which is produced by (1) thickening of the wall ; (2) accumalations of Filuid ; and (3) development of gas from fermentation within the loep. This condition he produced experimentally by ligation of the mesenteric vessels; after a ligature of the intestine on the proximal side of the portion in which the circulation was obstructed, the distal end being free, still the intestine dilated. This has great bearing in all operations of intestinal approximation, as it accounts for the paralysis and distension occurring where a greater portion of the mesenteric side of the bowel is removed than on the convex side. It will be admitted that a definite diagnosis of the pathological condition producing the obstruction may not be possible, but that does not prevent us from operating. Waiting will not help the patient, or even assist in making the dagnosis, and every hour lessens the patient's resistance and increases local destruction or necrosis. Still, every day we meet with cases in which this policy of procrastination is pursued until the patient is moribund; finally, and unfortunately too often, we are forced to soperate under just these unfarourable circumstances. When we operate under these conditions, are we warranted in abandoning the search for the cause of obstruction and its removal, for the establishment of an artificial anus? I think not. Saltzman states that laparotomies for ileus, with removal of cause of obstruction, up to 1883 had a mortality of 71.3 per cent.; simple enterotomy, without removal of cause, 67.3 per cent. So hopeless were the results of laparotomies with the removal of cause, that such surgeons as Mikulicz, Schede, and Shoenborn, who were formerly strong advocates of this operation, abandoned it for simple enterotomy to relieve the obstruction. The indications for the latter operation they attempted to extend, even with a slight percentage in its iavour and the comparative certainty that a second operation would be necessary. The establishment of a fæoal fistala in this class of cases should be permitted only as a last resort in a profoundly collapsed patient, as we have now means at hand of removing the necrotic intestine and re-establishing its continuity in a much shorter time than a fæcal fistula can vee formed. The more acute the attack and persistent the pain, the more pronounced the collapse, the more probable the existence of complete strangulation and local necrosis. There is, however, no positive symptom of necrosis.

If the foregoing principles are adhered to the intrinsic dangers of intestinal obstruction can be reduced to roinimam.

The operation of lateral approximation (anastomosis proper) is distinctly a modern operation. as it was first suc cessfully performed by Von Hacker of Vienna in 1886 . It rapidly gained adherents on account of the great mortality following the operation of circular enterorrhaphy, and on account of the many mechanical devices that were brought forward about that time, which were intended to simplify che operation (such as the plates used so extensively ofy
Dr. Senn) and on account of the larger aperture that could be secured by the lateral suture. The objections to this operation after resection are: 1. The amount of time and labour required, as both ends have to be closed with two rows of sutures, each row at least an inch long, necessitating four inches of suturing for the end, no matter what method may subsequently be used for the lateral apposition; then the approximation plates, or button, are inserted. If the suture be used it is advised (Abbe) that an opening four inches long be made and around this be placed one row of Crerny and two rows of Lembert sutures; these rows will average about ten inches each, which, with the four inches of suturing required to close the ends, make thirty-four inches of suturing. For each inch four to six stitches are required, making a total of 136-204 stitches. An expert operator is able to do this operation in a comparatively short time, as Abbe has done; but how long would it take the average operator? 2. The danger of necrosis of the inverted portion at some point over this long epace, which objection is of very sraall moment. 3 . The donger that one of the many stitches might penetrate the thin mucosa, as they must all include the tunica propria upon which it rests and be exposed in the calibre of the bowel, producing st:tch abscess (as in W. Pindfleisch's cases). 4. 'The broad cicatricial mass and the oce-balf inch of surface-approximation produced by these three rows of sutures might be expected to contract, as there is no juxtaposition of the incised edges of the various coats of the intestine. This is shown in the case of Salzwedel, lateral approximation of intestine with suture, and still it is the only case we found of post-operative stenosis of the intestine, showing that it is of very rare occurrence.

End-to-side approximation.-There are two places in the intestinal tract where this operation should always be given the preference: 1 . In resection for cicatricial occlusion of the pylorus the end of the duodenum should be joined to the side of the stomach. 2. Resection of the cæcum in adults on account of the inequality of size of colon and ileum. In the first case the food is allowed to enter the intestine in about its normal position, and duodenal digestion progresses about the same as if no operation had been performed; of still greater importance is the fact that by this method the bile and pancreatic secretions are not permitted to enter the stomach and cause such grave disturbances as are reported to have occurred (Dr. Heinrich Braun). Indto-side approximation has no other legitimate place in surgery of the small intestine after resection.

End-to-end approximation. - I note from the reports of cases in the last six years 134 intestinal approximations by all methods, of which 49 were end-to-end, 61 side-to-side, and 24 in which the position of approximation was not stated. Of these, 44 cases were those of intestinal approximation for acute obstruction, with 15 deaths (mortality $34 \cdot 1$ per cent.) ; for chronic obstruction 43 cases, with 12 deaths (mortality $27 \cdot 9$ per cent.) ; making a total, for obstruction, of 87 cases, with 60 recoveries and 27 deaths (a mortality of 31 per cent.). The methods employed in acute obstruction were: suture 28 , deaths 10 (mortality 35.6 per cent.) ; mechanical means 13 , deaths 3 (mortality $23 \cdot 1$ per cent.); and suture with mechanical aid 3 cases, with 2 deaths (mortality 66.7 per cent.). The methods employed in cbronic obstruction were: sutare, 28 cases, 9 deaths (mortality $32 \cdot 1$ per cent.); mechanical means, 13 cases, 2 deaths (mortality 15.4 per cent.); and suture with mechanical aid, 2 cases, with 1 death (mortality 50 per cent.). The total number of intestinal approximations for clnsure of artificial anus was 35, with 6 deaths and mortality $17 \cdot 1$ per cent. Of these, 24 were cases of suture, with 5 deaths (mortality 40.8 per cent.); 7 were treated by mechanical means, with 7 recoveries; and 4 by suture with mechanical means, with one death (mortality 25 per cent.). End-to-end approximation of the bowel is the most desirable position, as it most closely approaches the natural condition. Surgeons have always regarded the end-to-end as the ideal approximation. This was only deviated from with the hope of reducing the great mortality that resulted from the methods heretofore employed. Has this deviation accomplished the desired result? Our statistics answer, No. In 49 cases of end-to-end approximation by all methods the above statistics show a mortality of 16.3 per cent., while in 66 lateral approximations by all methods the mortality was 31.8 per cent. The results of lateral approximation, therefore, show 2 mortality about 100 per cent. higber than that of end-to-end approximation. Paul remarks that "end-to-end is preferab'e to lateral union, if it can be obtained with equal safely." 'The objections 
raised to the circular suture are, firstly, the time consumed; secondly, the possibility of perforation in parts not covered with peritoneum (mesenteric attachment); thirdly, the large number of sutures, and the liability that some of them would go through the entire thickness of the bowel and produce peritoneal infection; fourthly, with the invagination method there is the additional danger of post-operative progression of the invagination, finally producing obstruction; and, fifthly, the danger of obstruction from contraction of the necessarily broad cicatrix. While these objections have force against the end-to-end approximation by suture, not one of them is valid against the use of the button.

The first objection raised to the button was that it. is a foreign body in the intestinal tract and might cause obstruction. This accident has not occurred in one of the sixty cases operated upon, nor has it taken place in over 300 experiments, nor will it occur except where there is a pathological contraction of bowel. The second objection, that the necrosis of the tissue within the clasp of the button would be a source of danger, is purely mythical, as the bowel is securely sealed, par excellence, by this method. Infection has not occurred in a single case. The third objection, that the small surface of apposition would not prove adequate in the protection of the peritoneal cavity, has as little basis as there is for believing that the wall of the intestine itself is not strong enough to protect the peritoneum, as pathologists show that the intestine at the seat of approximation can scarcely be differentiated from the normal intestine. I have received no information, nor do I know of a case in which there was a failure of union by the button, nor do I believe that such failure will occur except where there is a septic peritonitis due to infection from without. The contraction of three-eighths of an inch in the opening reported by Keen in his case is in unison with the contraction of the bowel from its previously distended condition, and should be expected under these circumstances; but there is no proof that the contraction would have continued to complete closure as occurred with the bone-plates. Two years have elapsed since the first operation with the button, in many others over a year, and in a still greater number a shorter period of time I have received recent reports on most of these cases, and stenosis has not occurred in a single one.

[Clinical synopses of 34 cases of intestinal approximation by means of Murphy's button were given, showing the results obtained in support of the writer's conclusions.]

Conclusions. - 1. The more rapidly the operation is performed the less the danger from shock. 2. The less the manipulation and exposure of the intestine, the less the danger of infection, post-operative paralysis, and adhesions. 3. The more uniform and continuous the pressure at approximation, the greater the assurance of adhesion, and the less the liability of infiltration. 4. A line of approximation is as good as half an inch. 5. Mechanical means in the last six years have produced better results than the suture in both lateral and end-to-end approximations. 6. The mortality in end-to-end approximation is much less than in lateral apposition, and it should always be given the preference. 7. The more perfect the juxtaposition of the various layers, the less the interposition of fibrous tissue and the more complete the regeneration across the line of union. 8. The more extensive the approximation surface, the larger the fibrous deposit, the greater the contraction. 9. The contraction with end-toend is less than with lateral approximation. 10. The juxtaposition of the similar histological layers of the wall of the intestine is an assurance against cicatricial contraction. 11. The modus operandi of the button is based upon the following principles : firstly, it retains apposition automatically - that is, without suture; secondly, nnion of tissue is produced at the line of pressure atrophy; thirdly, the pressure atrophy is produced by elastic pressure; fourthly, it produces juxtaposition of the edges of the same coats; fifthly, the union is accomplished by the smallest possible cicatrix and therefore must yield the least contraction of any operation ; and, sixtbly, the rapidity of application gives all the advantage that the saving of time can accomplish.

The following is the technique of insertion of the button.

1. Cholecystenterostomy. - An incision is made from the edge of the ribs, two inches to the right of and parallel to the median line, extending downwards three inches. The gall-bladder and duodenum are drawn into the wound; needle with fifteen inches of silk is inserted in the duodenum directly opposite the mesentery, and at a point near the head of the pancreas: a stitch is taken throngh the entire wall of the bowel, one-third the length of the incision to be made;

the needle is again inserted one-third the length of the incision, from its outlet, in a line with the first, and embracing the same amount of tissue as the first. A loop three inches long is beld here, and the needle is inserted in a similar manner, making two stitches parallel to the first in the reverse direction, and one-fourth of an inch from it, coming out at a point near the original insertion of the

FIG. 1.

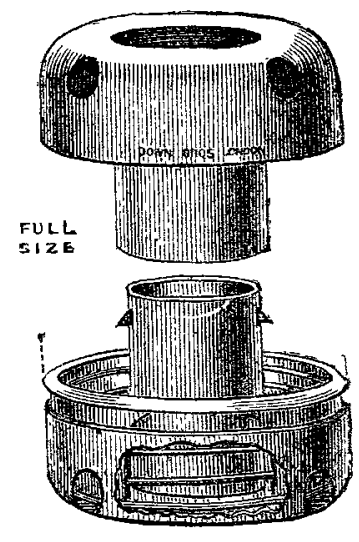

Anastomosis button.

needle. This forms the running thread, which, whed tightened, draws the incised edge of the bowel within the cup of the button. A similar running thread is inserted in the gall-bladder. An incision is now made in the intestine two-tbirds the length of the diameter of the button to be used; avoid cutting the running thread when making the incision. The button is slipped in, and the running thread is tied firmly around the central cylinder; an assistant holds the button in position with the bæmostatic forceps. An incision is now made in the gall-bladder, the same length as the one in tho intestine, between the rows of suture. The gall-stones

Fig. 2.

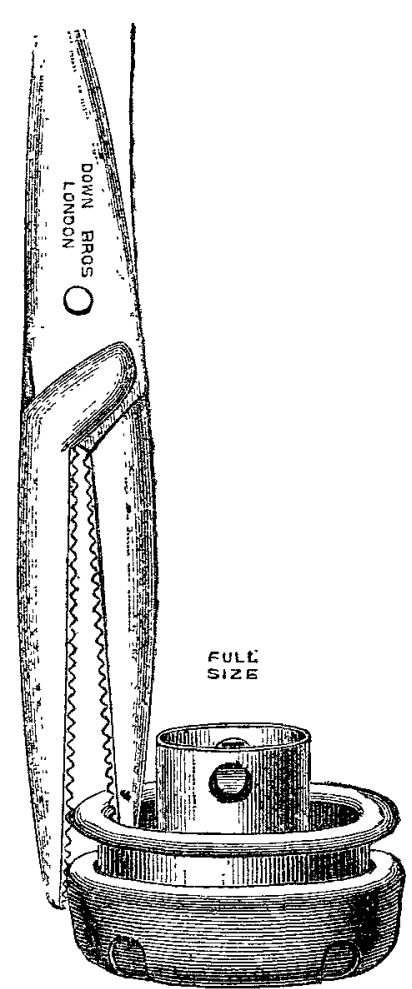

Showing method of holding elastic cup for insertion.

and fluid contents of the gall-bladder are removed, the button is inserted, and the running thread is tied. The forceps are then removed; the button is held between the fingers and slowly pressed together. (Fig. 4.) Note that the edge of the wound comes entirely within the clasp of the button before it is completely closed. A sufficient degree of pressure must be used to bring the serous surfaces of the gall-bladder and intestines firmly in contact and compress the tissues. The elastic pressure of the spring cup of the button produces a pressure atrophy of the tissues embraced 
by it and leaves an opening larger than the button. When the button is liberated it passes on through the bowel.

2. Pylorectomy.- Having drawn the pylorus into the abdominal wound, ligate the greater and lesser omenta and cat them off; then make a circular incision on a grooved

FIG. 4.

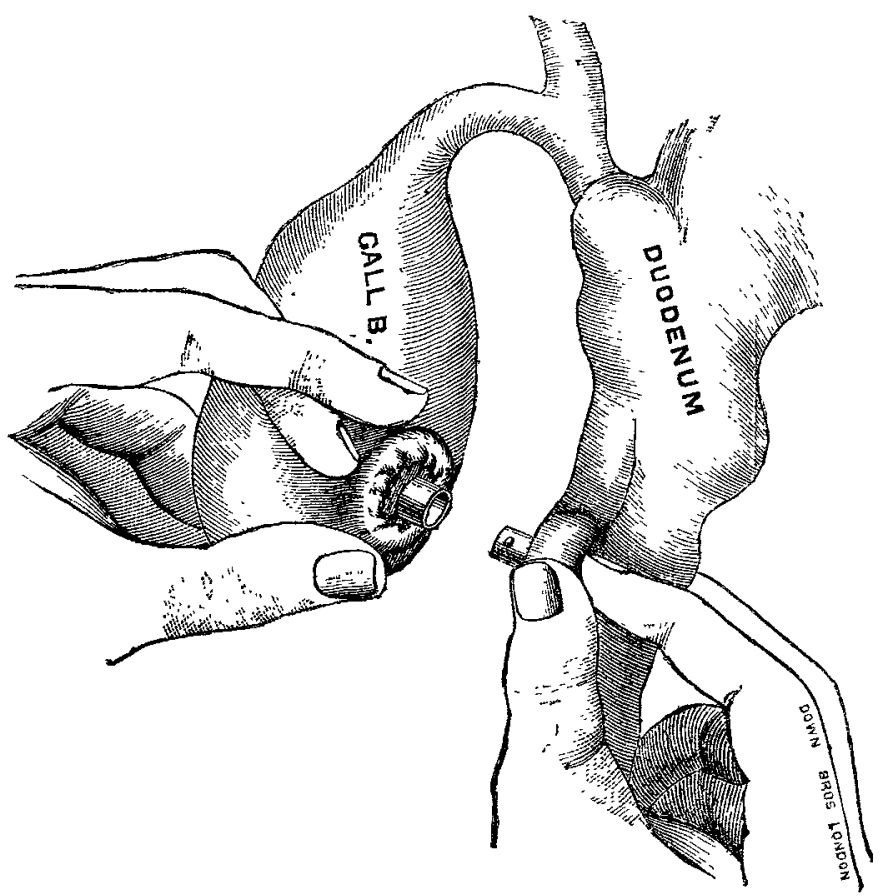

Showing method of holding parts while approximatins button in cholecystenterostomy.

director, with the scissors cutting through the muscular and serous coats down to the mucous coat, completely arcund the stomach where it is to be excised ; next denude the mucons membrane of its muscular and serous coats for half an inch in the direction of the fundus; excise the mucous membrane at this point ; close the mucous membrane with continuous catgut suture, and over this the serous and muscular coats with interrupted silk suture. The end of the stomach is

FIG. 5.

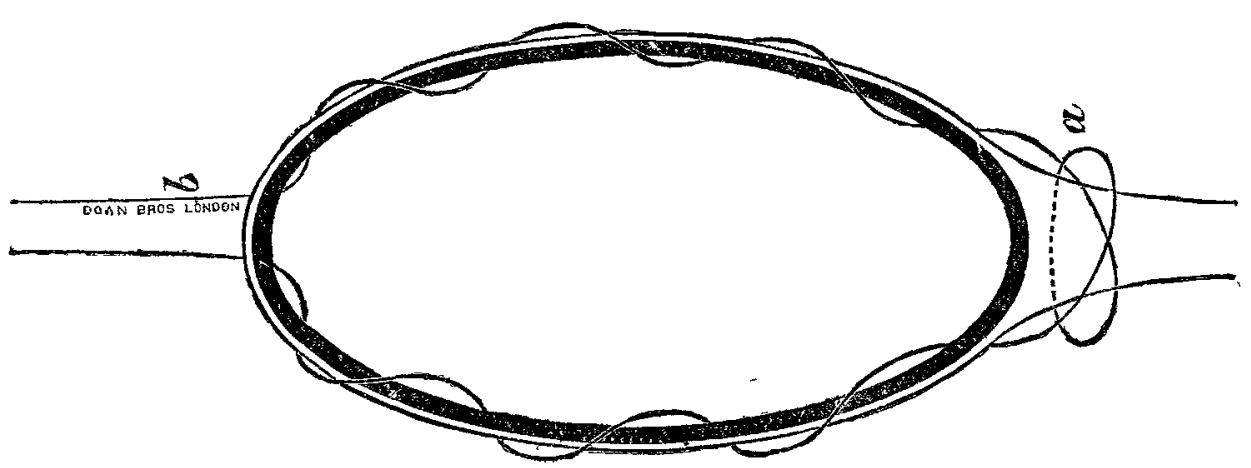

Suture of running thread as placed in end of bowel.

now completely closed. Excise the duodenum an inch and a half below the pylorus, insert a running thread as described below under the head of "end-to-end approximation," and place the male half of the button in position. The female half of the button is inserted in the posterior wall of the stomach $t$ two inches from the line of suture, in the same manner as it is inserted in the gall-bladder. The forceps are removed, and the button is pressed together. The end of the duodenum is then joined to the posterior wa l of the stomach. The button liberates itself and passes on through the bowel.

3. End-to-end approximation.-The intestine is cleared of its contents, and the intestinal compression clamps are placed in position. The mesentery of the portion to be excised is ligated ; the intestine is excised; a running tbread is placed in position by a top stitch along the excised $\epsilon$ dge, beginning opposite the mesentery and continuing down to the mesentery, one return over-stitch is taken at the mesentery (see Fig. 5, a); and then the top stitch is continued up the opposite side to the starting point ; this constitutes the "puckering string" and when tied around the stem of the button, which is then inserted, draws the cut edge within the clasp. Particular attention should be given to the return over-stitch at the mesentery, so that both layers of the peritoneum overlap. The other balf of the button is inserted in the srime manner, and the button is then pressed together. (Fig. 6.)

FIG. 6.
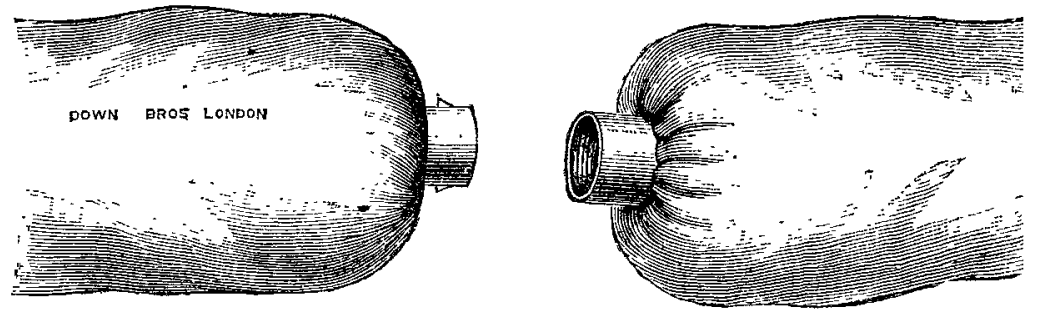

Button in end of bowel ready for cloning.

I advise the use of a special size button, one and a half inch in diameter, for the end-to-end approximation of the large intestine. It is more easily applied and has a larger central opening for the temporary passage of frecs.

4. Lateral approximation. - The button in the lateral approximation is inserted in the same manner as described in FIG. 7.

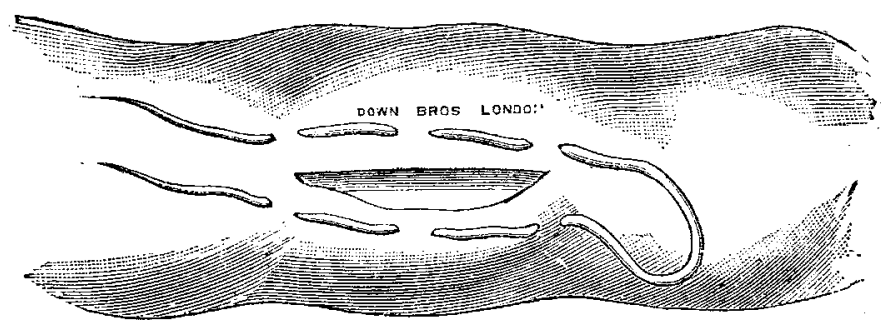

Showing method of introducing sutures in lateral approx ination, and relative size of incisior.

cholecystenterostomy. The spring cup is held down to the edge of the bowl by a forceps, as shown in FJg. 2 , while th $\Rightarrow$ hutton is being inserted. As soon as the button is in posi $i$ in change the hold by grasping it at the edge of the cylinder, and then tie the runxing thread. Do not make the incision too long, not more than two-thirds the diamever of the button; it will stretch. (Fig. 7.) Grasp the wall of the intestine at each end of the incision with tissue forceps while inserting the button. Do not cut the rumning thread when making the incision. Do sot enclose too much tissue in the suture. When closing the button be sure that the incised edge is always within the clasp of the button. Do not use a Lembert snture with the button; it is useless. It is not necessary to abrade the peritoneurn.

[Dr. Murphy appended numerons tables to his paper, showing the results obtained in intestinal approximation by various methods ; of these tables the most remarkable is one relating to the use of Murphy's button in cholecystenterostomy in non-malignant cases, whereof twenty-two patients all recovered.]

Presentations.-On the occasion of the distribution, at the town-hall, Batley, on Monday last, of certificates and medallions to the successfal members of the classes in connexion with the Batley Section of the Heavy Woollen Centre of the St. John Ambulance Association, Dr. Alfred Swann was presented with a handsome marble timepiece and pearl-handled knife, and at the same time $\mathrm{Mr}$. John Hall Woods was the recipient of a sum of money, which he generously handed to the mayor of the borough (who presided), with a request that it should go to the funds of the Batley Cottage Hospital.-Mr. H. T. Benson has been presented by the Market Deeping and District Ambulance Classes with a silver tankard and cigarette-case, inkstand, and silver knives. 